\title{
Situações de risco e vulnerabilidade em relação a possíveis transtornos mentais em crianças, adolescentes e mulheres
}

Risk and vulnerability situations in relation to possible mental disorders in children, adolescents and women

Situaciones de riesgo y vulnerabilidad en relación a posibles trastornos mentales en niños, adolescentes y mujeres

Mariana Rodrigues Silva

ORCID: https://orcid.org/0000-0002-9072-9174 Centro Universitário de Ciências e Tecnologia do Maranhão, Brasil

E-mail: rodriguessmaari@gmail.com

Isadora Sayonara Ferreira Coelho

ORCID: https://orcid.org/0000-0002-3300-6244

Centro Universitário de Ciências e Tecnologia do Maranhão, Brasil

E-mail: isaaebella3006@gmail.com

Ruth Lany Barros Araújo

ORCID: https://orcid.org/0000-0003-4981-1165

Centro Universitário de Ciências e Tecnologia do Maranhão, Brasil

E-mail: ruthlany@hotmail.com

Victor Emanoel da Silva Morais

ORCID: https://orcid.org/0000-0002-6752-5691

Centro Universitário de Ciências e Tecnologia do Maranhão, Brasil E-mail: victtor523@gmail.com

Cleiton Veloso Silva

ORCID: https://orcid.org/0000-0003-4831-7894

Centro Universitário de Ciências e Tecnologia do Maranhão, Brasil E-mail: cleitonveloso03@gmail.com

Thalita Cristina Oliveira Santos

ORCID: https://orcid.org/0000-0002-4284-7328

Centro Universitário de Ciências e Tecnologia do Maranhão, Brasil

E-mail: thalitacristina181@gmail.com

Vinícius Sampaio Melo do Nascimento

ORCID: https://orcid.org/0000-0001-6651-6112

Centro Universitário de Ciências e Tecnologia do Maranhão, Brasil

E-mail: vinirocksk8@gmail.com

George do Carmo Leão

ORCID: https://orcid.org/0000-0001-9106-1500

Centro Universitário de Ciências e Tecnologia do Maranhão, Brasil

E-mail: georgeleao13@gmail.com

Devydiana da Silva Salazar

ORCID: https://orcid.org/0000-0002-8766-5093

Centro Universitário de Ciências e Tecnologia do Maranhão, Brasil

E-mail: deyvdianadasilvas@gmail.com

Giovanna da Conceição dos Santos

ORCID: https://orcid.org/0000-0002-5683-6208

Centro Universitário de Ciências e Tecnologia do Maranhão, Brasil

E-mail: santosgio1702@gmail.com

Francisca Jorlanna da Silva Rocha

ORCID: https://orcid.org/0000-0003-2942-6585

Centro Universitário de Ciências e Tecnologia do Maranhão, Brasil

E-mail: silvajorlanna04@gmail.com

Luís Filipe Santos da Silva

ORCID: https://orcid.org/0000-0001-6403-2632

Centro Universitário de Ciências e Tecnologia do Maranhão, Brasil

E-mail: filipesantoscx@gmail.com

Felipe Delmiro dos Santos

ORCID: https://orcid.org/0000-0002-3983-2950

Centro Universitário de Ciências e Tecnologia do Maranhão, Brasil E-mail: felipedelmiro321@gmail.com 


\author{
Eudilene da Silva Mesquita \\ ORCID: https://orcid.org/0000-0001-9894-0903 \\ Centro Universitário de Ciências e Tecnologia do Maranhão, Brasil \\ E-mail: eu.mesquitta@gmail.com \\ Josefa Delmiro dos Santos \\ ORCID: https://orcid.org/0000-0002-8970-368X \\ Centro Universitário de Ciências e Tecnologia do Maranhão, Brasil \\ E-mail: josefadelmiro172@gmail.com
}

\begin{abstract}
Resumo
A Organização Mundial de Saúde (OMS) destaca brevemente questões, em que uma a cada quatro pessoas desenvolvem transtornos mentais em seu período de vida. Buscando assim compreender a ocorrência de transtornos mentais em crianças, adolescentes e mulheres, o presente estudo objetivou analisar a ocorrência de transtornos mentais em crianças, adolescentes e mulheres em situações de risco ou vulnerabilidade bem como verificar as possíveis causas relacionadas a estes transtornos mentais e investigar os possíveis transtornos relacionados à criança, ao adolescente e à mulher. Para a realização desta pesquisa realizou-se um estudo transversal utilizando base de dados como BIREME e BVS, utilizando como fator de inclusão materiais publicados nos anos de 2015 a 2020 . Os resultados apontam relação entre a ansiedade pré-natal materna com problemas emocionais na prole posteriormente, logo em se tratando da exposição à violência e sua repercussão na saúde mental do adolescente, características emocionais, aspectos relacionados à vulnerabilidade e uso de drogas na adolescência são fatores para desenvolvimento de transtornos mentais, já com relação à população infantil houve apenas resultados encontrados de materiais dessa temática relacionados às crianças autistas. Em síntese os estudos apontam o quanto questões de vulnerabilidade e situações de risco em crianças, adolescentes e mulheres colaboram consequentemente para quadros de adoecimento mental nos mesmos. Por fim, conclui-se que há uma necessidade de ampliação de estudos sobre o tema abordado ao qual, dessa forma contribuirá para melhores resultados e, consequentemente compreensão acerca os transtornos mentais relacionados à criança, adolescente e mulher.
\end{abstract}

Palavras-chave: Criança; Adolescente; Mulher; Risco; Mental.

\begin{abstract}
World Health Organization (WHO) briefly highlights issues in which one in four people develop mental disorders in their lifetime. In order to understand the occurrence of mental disorders in children, adolescents and women, this study aimed to analyze the occurrence of mental disorders in children, adolescents and women in situations of risk or vulnerability, as well as to verify the possible causes related to these mental disorders and investigate possible disorders related to children, adolescents and women. To carry out this research, a cross-sectional study was carried out using databases such as BIREME and BVS, using as an inclusion factor materials published in the years 2015 to 2020. The results show a relationship between maternal prenatal anxiety and emotional problems in the offspring later, when it comes to exposure to violence and its repercussions on the mental health of adolescents, emotional characteristics, aspects related to vulnerability and drug use in adolescence are factors for the development of mental disorders, as for the child population there were only results found of materials on this theme related to autistic children. In summary, the studies show how much vulnerability issues and risk situations in children, adolescents and women consequently collaborate to mental illness in them. Finally, it is concluded that there is a need to expand studies on the topic addressed which, in this way, will contribute to better results and, consequently, understanding about mental disorders related to children, adolescents and women.
\end{abstract}

Keywords: Child; Adolescent; Women; Risk; Mental.

\title{
Resumen
}

Incluir La Organización Mundial de la Salud (OMS) destaca brevemente los problemas en los que una de cada cuatro personas desarrolla trastornos mentales a lo largo de su vida. Con el fin de comprender la ocurrencia de trastornos mentales en niños, adolescentes y mujeres, este estudio tuvo como objetivo analizar la ocurrencia de trastornos mentales en niños, adolescentes y mujeres en situaciones de riesgo o vulnerabilidad, así como verificar las posibles causas relacionadas con estos. trastornos mentales e investigar posibles trastornos relacionados con niños, adolescentes y mujeres. Para la realización de esta investigación se realizó un estudio transversal utilizando bases de datos como BIREME y BVS, utilizando como factor de inclusión materiales publicados en los años 2015 a 2020 . Los resultados muestran una relación entre la ansiedad prenatal materna y los problemas emocionales en el descendencia luego, cuando se trata de la exposición a la violencia y sus repercusiones en la salud mental de los adolescentes, las características emocionales, los aspectos relacionados con la vulnerabilidad y el consumo de drogas en la adolescencia son factores para el desarrollo de los trastornos mentales, ya que para la población infantil solo se obtuvieron resultados encontrado de materiales sobre este tema relacionados con los niños autistas. En resumen, los estudios muestran cuánto los temas de vulnerabilidad y las situaciones de riesgo en niños, adolescentes y mujeres colaboran consecuentemente con la enfermedad mental en ellos. Finalmente, se concluye que existe la necesidad de ampliar los estudios sobre el tema abordado que, de esta manera, contribuyan a mejores resultados y, en consecuencia, al entendimiento sobre los trastornos mentales relacionados con niños, adolescentes y mujeres.

Palabras clave: Niño; Adolescente; Mujeres; Riesgo; Mental. 


\section{Considerações Iniciais}

Inicialmente, torna-se evidente ressaltar que há um imenso e vasto campo para a designação do estado de transtorno mental em crianças, adolescentes e mulheres aos quais se apresentam em situações de risco e vulnerabilidade. Desse modo, em algumas situações existem fatores norteadores para que um indivíduo possa chegar à um estado de adoecimento caracterizado por um determinado tipo de transtorno mental. Em contrapartida, para que possa existir a saúde individual e coletiva, torna-se necessário o acesso ao saneamento básico, alimentação saudável, políticas de imunização, acesso ao lazer, educação, saúde e entre outros fatores (Tavares, et al., 2019).

Além disso, a Organização Mundial de Saúde (OMS) destaca brevemente questões em que de uma a cada quatro pessoas desenvolvem transtornos mentais em seu período de vida. Desse modo, destacam-se dentre os transtornos mentais mais comuns, sintomas depressivos, ansiedade e entre outros fatores aos quais destaca-se um número mais elevado desses casos em mulheres comparado à população masculina (Senicato, et al., 2018).

Por outro lado, a OMS destaca também, relatórios acerca dos transtornos mentais e seus aspectos casuais para o aparecimento dos mesmos, em que é relatado surgimento de transtornos na infância e adolescência, que são comuns, e que esta população representa aproximadamente alta taxa de prevalência em transtornos relacionados à saúde mental, aos quais estão inseridos dentro desse contexto transtornos do desenvolvimento psicológico, transtornos de comportamentos e emocionais (Thiengo, et al., 2014).

As psicopatologias e os efeitos adversos encontrados no cérebro são considerados significativamente que o indivíduo passou por momentos de maus-tratos durante um período da infância, e isso torna um grau de risco bastante elevado para o desenvolvimento de transtornos mentais, como por exemplo, o de humor e o uso de substâncias impróprias. Os transtornos podem variar de ao grau de exposição à violência e/ou aos maus-tratos. (Kirsch, et al., 2021).

Por conseguinte, acerca o desenvolvimento de transtornos mentais na população, trona-se importante salientar que para compreender esse fator saibamos as possíveis causas aos quais a mulher represente uma maior vulnerabilidade em que estão associadas geralmente à fatores do sistema endócrino durante o período pré-menstrual, pós-parto, menopausa, fatores sociais como a desigualdade de gênero, fatores biológicos e entre outros (Senicato, et al., 2018).

\subsection{Transtornos Mentais Relacionados à Criança}

Os transtornos mentais na infância comprometem o desenvolvimento neurológico do indivíduo e cerca de $20 \%$ das crianças de países subdesenvolvidos sofrem de transtornos mentais em que estes são considerados irrelevantes. O mito da caverna de Platão faz referência a pessoas que se recusavam a observar a realidade em virtude do medo de sair de sua zona de conforto, em contrapartida podemos observar nesta problemática que uma grande parte da sociedade se recusa a observar a importância da saúde mental infantil não tratando as crianças como um todo. Além disso, pesquisar a prevalência das alterações comportamentais e sentimentais na criança é a primeira coisa a fazer-se para entender o que está acontecendo e determinar as causas que levaram ao surgimento desses problemas, podendo assim trata-los (Bach, et al., 2019).

Existe uma alta prevalência de transtornos de atenção e hiperatividade em meninos e transtornos emocionais em meninas, há também uma alta relação entre a presença de transtornos mentais nos pais biológicos e consequentemente com os transtornos nos filhos, além disso, os estudos mostraram que nos primeiros anos do ensino fundamental há uma grande prevalência de transtornos emocionais e comportamentais, que podem ser mais facilmente identificados no ambiente escolar. Ademais, distúrbios como depressão e outros transtornos presentes na figura materna influencia diretamente no psicológico da criança pois o relacionamento entre mãe e filho vai induzir e trazer consequências no seu convívio social (Bach et al., 2019).

Segundo Rees, Channon e Waters (2019) há casos em que a prevalência de transtornos durante o período de pré-natal, parto e puerpério podem impactar em resultados adversos no sistema psicológico da criança ocasionando uma gama de 
distúrbios comportamentais, cognitivos e neurofisiológicos. Dessa maneira, existem vários fatores que favorecem o desenvolvimento de transtornos mentais em crianças, logo os resultados evidenciaram que a ansiedade puerperal também está ligada aos transtornos nos filhos que tinham idade variada de 2 a 5 anos.

Segundo Monteiro, et al (2020) Os transtornos mentais se tornaram um grande desafio para os serviços de saúde geralmente, esse sofrimento psíquico é manifestado por sinais de ansiedade e depressão que é um problema de saúde que acomete crianças e adolescentes em todo mundo que para os profissionais da saúde tem grande problemas em diagnosticar e associar as condições de risco para que crianças e adolescentes desenvolvam e adoeçam mentalmente.

Além disso, a superproteção e o controle psicológico podem gerar transtornos mentais graves, e não se deve confundir controle comportamental com controle psicológico, ambos têm significados e estratégias diferentes de ensino. Controle psicológico está ligado à ocorrência de transtornos mentais internalizastes e controle comportamental está ligada diretamente como a criança irá se portar diante de grupos ou pares de convivência (Lins \& Alvarenga, 2015).

Em contrapartida, o desenvolvimento de transtornos mentais, assim como distúrbios alimentares da criança está fortemente relacionada ao cuidador(a), em casos de transtorno obsessivo compulsivo relaciona-se à fobias alimentares e a referência ou expressão que a criança faz sobre determinado alimento, assim sendo o cuidador acaba que inibe aquele tipo de alimento essencial para a criança que entretanto passa a desenvolver transtorno obsessivo compulsivo, pois vai usar da seletividade de alimentos. Deste modo é um transtorno mental que precisa de investigação pediátrica juntamente com psicólogo e psiquiatra (Bozzini, et al., 2019).

\subsection{Transtornos Relacionados ao Adolescente}

A adolescência destaca-se por ser um período de transição e repleto de mudanças aos quais ajudam para posteriormente a formação da identidade adulta e individual daquele indivíduo, dessa maneira na obra "Saúde mental e cuidado de enfermagem em psiquiatria" retrata brevemente questões acerca dos transtornos mentais relacionados ao adolescente em que nesse contexto destaca-se a importância de se analisar possíveis situações de risco ou vulnerabilidade a aquele paciente adolescente que está sendo atendido, desse modo, os transtornos mais frequente a este grupo destacam-se a depressão, hiperatividade, transtorno de ansiedade e também, conduta (Tavares, et al., 2019).

De certo, em se tratando de possíveis fatores relacionados como possíveis causas de transtornos mentais em adolescentes, deve-se ressaltar os fatores biológicos cujo referem-se frequentemente à anormalidades no sistema nervoso central daquele indivíduo; fatores psicossociais em que associa-se a alterações na vida familiar daquele indivíduo ocasionando um possível estressor; fatores genéticos ligados diretamente à herança genética de um possível transtorno caso haja um histórico familiar e fatores ambientais que se relacionam à problemas em comunidade como baixa renda, violência e etc (Tavares, et al., 2019).

É notório esclarecer que a família se classifica como um viável fator protetor constituindo situações saudáveis e consequentemente reduzindo questões acerca transtornos relacionados à esta fase de vida, ou mesmo possa se manifestar como um risco em que expõe a situações de violência ou uso de drogas no ambiente daquele adolescente. Desse modo, pelo fato deste período de transição se apresentar com oscilações/turbilhão de sentimentos e hormônios e possivelmente uma fase em busca de experimentos e aprendizado, é notável que adolescentes, com família que se encaixa como um risco, encontram-se vulneráveis ao uso de drogas, o que pode surtir um efeito de mascarar situações conflitantes e consequentemente possa caracterizar um problema à aquele adolescente em que, ao que se sabe o uso inadequado de substâncias durante a adolescência tem sido associado à alterações na estrutura cerebral e entre outras repercussões negativas no campo social na vida daquela pessoa (Luciano, et al., 2017). 
A adolescência é uma fase da vida que os indivíduos têm hábitos que podem ser de riscos e que podem leva-los ao suicídio, além disso, vários fatores podem influenciar adolescentes a terem comportamentos suicidas e esses fatores são encontrados na literatura como: família, orientação sexual, baixa renda, histórico familiar, solidão, uso de substâncias psicoativas, entre outros. E assim, desencadeando um problema de saúde mundial, é necessário saber os fatores das causas entre jovens para prevenir esse problema (Sousa, et al., 2019).

Nessa fase que o adolescente está descobrindo coisas novas, conhecendo novas amizades, aprendendo novos caminhos e nesse período que o conhecimento se amplia principalmente em relação ao uso das drogas ilícitas como maconha, cocaína, opióides, tabaco, cafeína, benzodiazepínicos e anfetaminas, entre outros. Os adolescentes em situação de risco ou vulnerabilidade estão mais susceptíveis a realizarem o uso de substâncias psicoativas, buscando tentar esquecer os problemas sejam eles familiares, sociais ou econômicos, que consequentemente podem levar ao desenvolvimento de transtornos mentais como ansiedade, depressão, transtorno bipolar, esquizofrenia entre outros. O uso abusivo de substâncias psicoativas é como uma fuga da realidade (Barbosa, et al., 2020).

De acordo com Patias, Silva e Aglio (2016), A violência pode-se encaixar diretamente como uma das causas à transtornos mentais em adolescentes em que pode trazer efeitos negativos aos quais podem interromper ou atrasar o desenvolvimento social, cognitivo, emocional e, potencializar risco a psicopatologias. Dessa maneira, entre os principais transtornos mentais associados à violência estão: Estresse Pós-Traumático, transtornos de ansiedade, transtornos de humor e transtornos de personalidade. Em suma, estudos literários identificaram alta prevalência de exposição à violência em vários contextos do desenvolvimento do adolescente sejam estas de tentativas de suicídio, violências físicas, interpessoal e sexual aos quais se relacionam consequentemente para um desenvolvimento negativo à saúde mental do adolescente e que, em suma a junção entre juventude e violência quando não leva a morte, traz consigo resultados como redução da expectativa de vida, redução do potencial produtivo e comprometimento de saúde do adolescente.

\subsection{Transtornos Relacionados à Mulher}

De acordo com Barbosa, Souza e Freitas (2015) É evidente dificuldades desse grupo de mulheres em cuidado com a saúde mental, já que na maioria das vezes são ignoradas pela sociedade e vistas como loucas. Além disso, o estudo mostra situações reais de completo sofrimento e abandono por parte dessas mulheres e como é importante o papel do profissional de saúde bem preparado para atender as mesmas. Desse modo, o estudo realizado somente com mulheres em situações de violência sexual e transtornos mentais mostra a vulnerabilidade das mesmas em que todas estão em situações de pobreza, ou extrema pobreza e com baixo nível de escolaridade. Em suma, todo esse contexto faz com que o atendimento seja mais difícil em que a maioria não tem noção de suas reais condições de saúde e não indo procurar à ajuda especializada.

Em estudos literários foram identificados que uma parte significativa das gestantes apresentaram sugestão de adoecimento mental, o qual se encontra associado a estar solteira, baixo nível de escolaridade, não planejou a gestação e possui hipertensão arterial sistêmica. Além disso, estes resultados despertam a atenção as questões de saúde mental durante o acolhimento e consulta de enfermagem durantes pré-natal natal, olhando para gestante o como um todo e conseguindo detectar fatores que aumentam suas chances para ocorre adoecimento mental ampliara a capacidade do profissional para o cuida da enfermagem, e em consequências, devem tornar mais assertivas as demais fases do processo de assistência de enfermagem (Guimarães, et al., 2019).

As mulheres puérperas também são um importante grupo de risco para o desenvolvimento de transtornos mentais, dentre eles encontramos a depressão. O pós-parto é um período de grandes mudanças fisiológicas, biológicas, sociais e emocionais, o que favorece o aparecimento das doenças mentais. Os fatores de risco que influenciam significativamente são baixa renda, gravidez não planejada, ansiedade na gravidez, violência doméstica, eventos estressantes, falta de apoio familiar e 
social além da idade mais jovem. Esses fatores favorecem o aparecimento da depressão puerperal que vai afetar o dia a dia daquela mulher, a relação entre o binômio mãe-bebê, além de influenciar negativamente no crescimento e desenvolvimento posterior da criança (Duko, et al., 2020).

A ocorrência de TMC (transtornos mentais comuns) são mais prevalentes em mulheres, devido a uma grande associação de várias atividades, como trabalhos domésticos, os cuidados com a família, e a carreira profissional. E essas associações requerem uma grande demanda de trabalho, tornando as mulheres mais suscetíveis ao desenvolvimento de transtornos, entre os mais comuns, a depressão e ansiedade. Um estudo mostrou, que no período gestacional a predisposição de desenvolvimento de TMC (transtornos mentais comuns), são ainda mais recorrentes em virtude das alterações hormonais, as condições socioeconômicas, nível de escolaridade, renda familiar, idade e estado civil. Os critérios do planejamento da gravidez e a falta do apoio familiar, como a ausência do companheiro, também são fatores determinantes que contribuem para o desenvolvimento de transtornos mentais (Lucchese, et al., 2017).

De acordo com a Organização Mundial de Saúde (OMS), durante a vida, uma em cada quatro pessoas tendem a desenvolver algum tipo de transtorno mental, apresentando quadros de ansiedade e outros sintomas somáticos não característicos, sendo o transtorno mental comum (TMC) mais recorrente em mulheres. A maior prevalência de transtornos se dá aos diversos fatores que englobam esse grupo, aspectos fisiológicos como as alterações do sistema endócrino que ocorrem desde o período pré-menstrual a menopausa, aspectos sociais como a desigualdade de gênero e a violência, além de fatores psicossociais e socioeconômicos (Azevedo, et al., 2018).

Dentre os fatores de risco para o desenvolvimento de transtornos mentais está a violência por parceiro íntimo (VPI), situação vivida por cerca de 50\% das mulheres no Brasil. Essa condição ainda acarreta o surgimento e a evolução de outros transtornos como o transtorno mental comum (TMC), quadros de ansiedade e depressão. A prevalência de TMC entre mulheres que sofreram todos os tipos de violência é de $62,9 \%$ e 30,6\% às que sofreram apenas de violência física (Mendonça \& Ludemir, 2017).

Inclusive, vale ressaltar que há um número exuberante de mulheres vítimas de violência com adoecimento mental, pois as mulheres vítimas de agressão tais como: violência de gênero, física, sexual, mental, entre outras, são também populações de risco para a constituição de fatores formadores do adoecimento mental, afetando a vida e a saúde dessas mulheres. A ansiedade e a depressão são as mais prevalentes nesse contexto assim, deixando claro as dificuldades e as vulnerabilidades que esse grupo sofre diante da não intervenção adequada a tais situações. Portanto, cabe uma reflexão sobre como os profissionais de saúde devem atuar para atenuar o acontecimento desse grande problema de saúde pública (Medeiros \& Zannello, 2018).

Os fatores de risco e vulnerabilidade para desenvolvimento de transtornos mentais em crianças, adolescentes e mulheres estão presentes na sociedade cotidianamente de formas variadas e que podem desencadear sérios problemas a estas populações. Além disso, estes transtornos podem estar associados a diversos fatores que podem ser ambientais, sociais ou biológicos.

A princípio, os questionamentos a respeito desse projeto foram: Como ocorre o desenvolvimento de transtornos mentais em crianças e adolescente? Qual a possibilidade de mulheres em situações de risco desenvolver transtornos mentais? Diante disso torna-se necessário compreender a ocorrência de transtornos mentais em crianças, adolescentes e mulheres, além de analisar a ocorrência de transtornos mentais em crianças, adolescentes e mulheres em situações de risco ou vulnerabilidade e verificar as possíveis causas relacionadas a estes transtornos mentais em crianças, adolescentes e mulheres a fim de investigar os possíveis transtornos relacionados à criança, ao adolescente e à mulher.

Eventualmente, há uma grande prevalência de casos de transtornos mentais relacionados à criança, ao adolescente e, também a mulher. Desse modo, torna-se evidente, portanto o quanto é relevante e desafiador essa temática visto que, traz 
consigo contribuições tanto para o conhecimento científico das pessoas em geral como para a promoção, prevenção e recuperação da saúde mental dos indivíduos, pois o referido projeto traz consigo informações e dados importantes sobre a saúde mental e transtornos mentais relacionados às crianças, adolescentes e mulheres que se encontram em situações de risco ou vulnerabilidade e, desse modo, auxilia na melhoria da qualidade dos programas de atenção a saúde mental, a educação em saúde e consequentemente no progresso da saúde global.

\section{Metodologia}

Para a realização desta pesquisa buscou-se compreender a ocorrência de transtornos mentais em crianças, adolescentes e mulheres, além disso, buscou-se também analisar a ocorrência dos mesmos e, verificar as possíveis causas sempre investigando os transtornos mentais relacionados a estes grupos. Desse modo, procurou-se entender a forma em que acontece esses transtornos e os devidos fatores que influenciaram a ocorrência deles.

Além disso, o presente trabalho trata-se de um estudo transversal ao qual avalia a frequência da ocorrência de transtornos mentais, bem como os efeitos causados por estes transtornos em crianças, adolescentes e mulheres em que se utilizou a coletas de materiais entre 2015 e 2020. É importante ressaltar, que foram utilizadas a estratégia PICO nos problemas de pesquisa ao qual analisaram-se P: Crianças, adolescentes e mulheres; I: situações de risco ou vulnerabilidade; C: Ausência de transtornos; O: se há relação de possíveis transtornos mentais em crianças, adolescentes e mulheres em situações de risco ou vulnerabilidade.

Logo, foi realizada pesquisas no DeCS para localizar os descritores da pesquisa em que se evidenciou como descritores:/. Em seguida, foi realizada uma pesquisa avançada na base de dados Bireme com utilização dos operadores boleanos: (crianças ) AND (situação OR risco) AND (transtorno OR mental) AND ( type_of_study:("systematic_reviews") AND la:("pt")) AND (year_cluster:[2015 TO 2020]); (adolescente) AND (situação OR risco OR vulnerabilidade) AND (transtorno OR mental) AND ( type_of_study:("systematic_reviews") AND la:("pt")) AND (year_cluster:[2015 TO 2020]); (mulher) AND (vunerabilidade OR situação OR risco) AND (transtorno OR mental) AND ( type_of_study:("systematic_reviews")) AND (year_cluster:[2015 TO 2020]).

Além disso, foi efetuada uma pesquisa também, na biblioteca virtual BIBLIOTECA A ao qual foi selecionado 1 livro E-book e, em contrapartida, em relação às pesquisas realizadas na Bireme foram selecionados um total de 10 materiais.

Em suma, as referências selecionadas foram utilizadas como embasamento para os referenciais teóricos ao qual buscou-se fatores de compreensão de como ocorre transtornos mentais em crianças, adolescentes e mulheres e, relacionando este fator à indivíduos destes grupos que se encontram em vulnerabilidade. Por conseguinte, concluída a pesquisa em busca dos referenciais teóricos, deram-se as atividades de síntese acerca as pesquisas.

\section{Resultados}

Selecionaram-se 10 materiais para a análise desse projeto aos quais foram publicados nos últimos cinco anos (2015, 2016, 2017, 2018 e 2019). Outrora, quanto aos dados categóricos foram analisados transtornos relacionados em crianças, adolescentes e mulheres.

\subsection{Criança}

Quanto aos transtornos obsessivos compulsivos (TOC) em crianças com dificuldades alimentares, pode-se analisar que nos materiais inclusos para esta pesquisa evidenciaram questões em que há uma escassez de publicações sobre esse tema. Em contrapartida, foram coletados dados de indivíduos adultos com e sem seletividade de alimentos relacionando esse fator ao TOC, em que é importante ressaltar a analogia de que indivíduos adultos com TOC e seletividade alimentar posteriormente 
acarretaram essa característica desde a infância. Outrora, foram analisados fatos, em que indivíduos com as características citadas anteriormente, possuem uma propensão de sintomatologias de ansiedade, nojo e rigor no comportamento alimentar. Ademais, voltando para a população de crianças houve um número de resultados encontrados de materiais dessa temática apenas relacionados às crianças autistas. Em contrapartida, segue abaixo os resultados conforme o Quadro 1.

Quadro 1 - Resultados de TOC em indivíduos e crianças com autismo relacionados a dificuldades alimentares.

\begin{tabular}{|l|l|}
\hline \multicolumn{1}{|c|}{ AUTORES E ANO } & \multicolumn{1}{c|}{ RESULTADOS } \\
\hline Bozzini, et al., 2018. & $\begin{array}{l}\text { Sintomas de TOC elevados relacionados à uma propensão de sintomas depressivos } \\
\text { posteriormente. }\end{array}$ \\
\hline & Rigor no comportamento alimentar maior em indivíduos com TOC. \\
\hline & Crianças ansiosas possuem uma maior probabilidade de dificuldades alimentares. \\
\hline
\end{tabular}

Fonte: Artigo analisado (2018).

O Quadro 1 demonstra os resultados do estudo de Bozzini, et al., 2018. Apontando a relação do desenvolvimento de transtorno como a depressão em indivíduos portadores de TOC, e a rigorosidade alimentar de indivíduos com TOC, além de apontar dificuldades alimentares em crianças ansiosas.

A princípio, acerca dos problemas internalizantes em crianças, os materiais inclusos descreveram correlações desses problemas com alguns fatores aos quais estão descritos no Quadro 2.

Quadro 2 - Correlações entre os problemas internalizantes.

\begin{tabular}{|l|l|l|}
\hline \multicolumn{1}{|c|}{ AUTORES E ANO } & \multicolumn{1}{|c|}{ CORRELAÇÕES } & \multicolumn{1}{|c|}{ RESULTADOS OBTIDOS } \\
\hline Lins \& Alvarenga, 2015. & $\begin{array}{l}\text { Problemas internalizantes correlacionados } \\
\text { negativamente à idade dos pais e a quantidade } \\
\text { de irmãos da criança. }\end{array}$ & $\begin{array}{l}\text { Síndrome da reatividade emocional relacionada } \\
\text { com aspectos negativos; correlação negativa de } \\
\text { ansiedade/ depressão e; queixas somáticas. }\end{array}$ \\
\hline & $\begin{array}{l}\text { Análise e comparação de diferenças nos } \\
\text { escores de crianças com problemas }\end{array}$ & $\begin{array}{l}\text { Os escores de depressão e ansiedade foram } \\
\text { maiores para crianças em que os pais não } \\
\text { residem juntos, comparados à crianças que os } \\
\text { pais moram na mesma residência }\end{array}$ \\
& $\begin{array}{l}\text { internalizantes, relacionados a fatores } \\
\text { sociodemográficos como o sexo da criança, } \\
\text { ocupação da mãe e coabitação parenteral. }\end{array}$ & \\
\hline
\end{tabular}

Fonte: Artigo analisado (2015).

O Quadro 2 demonstra os resultados do estudo de Lins e Alvarenga, 2015. Onde encontraram-se síndrome da reatividade emocional relacionada com ansiedade/depressão, que foram ocasionadas por problemas internalizantes como a idade dos pais e a quantidade de irmãos da criança. Ele também aponta maior desenvolvimentos de transtornos como depressão e ansiedade em crianças de pais separados.

Com base em teóricos referenciados para o embasamento deste trabalho foram evidenciadas questões acerca a ansiedade pré-natal materna relacionada à problemas emocionais na prole posteriormente (Quadro 3). 
Quadro 3 - Ansiedade pré-natal materna relacionada à problemas emocionais na criança.

\begin{tabular}{|l|l|l|}
\hline \multicolumn{1}{|c|}{ AUTORES E ANO } & $\begin{array}{c}\text { ANSIEDADE PRÉ-NATAL E } \\
\text { AGRAVANTES }\end{array}$ & \multicolumn{1}{c|}{ RESULTADOS } \\
\hline Rees, et al., 2019. & Mães com ansiedade pré-natal & $\begin{array}{l}\text { Filhos com níveis mais elevados de problemas } \\
\text { emocionais comparado aos filhos de mães sem um } \\
\text { quadro de ansiedade pré-natal. }\end{array}$ \\
\hline & $\begin{array}{l}\text { Onsiedade social materna da ansiedade social materna foi positivo para } \\
\text { crianças com apego seguro e, negativo para crianças } \\
\text { com apego inseguro. }\end{array}$ \\
\hline pré-natal & $\begin{array}{l}\text { As filhas de mães com baixo AVC apresentaram } \\
\text { problemas emocionais crescentes, enquanto esse efeito } \\
\text { não foi observado nas meninas, cujas mães pertenciam } \\
\text { ao grupo com alto curso, nem nos meninos. }\end{array}$ \\
\hline
\end{tabular}

Fonte: Artigo analisado (2019).

O Quadro 3, descreve os estudos de Rees, et al., 2019. Onde encontra-se maior probabilidade do filho desenvolver problemas emocionais quando a mãe tem ansiedade pré-natal; também demonstra que crianças com maior apego à mãe tende a sentir um efeito maior da ansiedade que a mesma possui.

\subsection{Adolescente}

Quanto à exposição à violência e sua repercussão na saúde mental do(a) adolescente, características emocionais, aspectos relacionados à vulnerabilidade e uso de drogas na adolescência, pode-se observar diversos resultados cujo estão evidenciados no Quadro 4.

Quadro 4 - Resultados obtidos de acordo com os estudos selecionados e principais considerações.

\begin{tabular}{|c|c|c|c|}
\hline TÍTULO & AUTOR & ANO & PRINCIPAIS CONSIDERAÇÕES \\
\hline $\begin{array}{l}\text { Exposição de Adolescentes à } \\
\text { Violência em Diferentes } \\
\text { Contextos: Relações com a Saúde } \\
\text { Mental }\end{array}$ & $\begin{array}{ll}\text { Patias } & \& \\
\text { Silva } & \& \\
\text { Aglio. } & \end{array}$ & 2016 & $\begin{array}{l}\text { A alta prevalência de exposição a violência em diversos contextos do } \\
\text { desenvolvimento da adolescência, resultou em consequências } \\
\text { negativas, como por exemplo: depressão, ansiedade, uso de álcool e } \\
\text { outras drogas, comportamento sexual de risco, sintomas agressivos, } \\
\text { desajustamento psicossocial, problemas externalizantes, ideação } \\
\text { suicida, situações de maior uso de armas e violência entre meninos, } \\
\text { transtorno de estresse pós-traumático (TEPT) e, problemas de } \\
\text { autoestima. }\end{array}$ \\
\hline $\begin{array}{l}\text { Perfil de adolescentes } \\
\text { vulnerabilidade para o uso de } \\
\text { álcool e outras drogas }\end{array}$ & $\begin{array}{l}\text { Tavares, } \\
\text { et al. }\end{array}$ & 2017 & $\begin{array}{l}\text { Dentre os resultados, observou-se que de um estudo com } 240 \\
\text { adolescentes com uma perda de } 16 \% \text { dessa população, quanto às } \\
\text { características emocionais maioria }(82,1 \%) \text { se considera feliz e, } \\
\text { quanto à vulnerabilidade para o uso de drogas estão relacionados à } \\
\text { saúde em que, percebe-se que o álcool é a substância com o maior } \\
\text { uso. Ademais, foram evidenciados o uso de maconha, dietilamida do } \\
\text { ácido lisérgico (LSD), tabaco, loló, cocaína, ecstasy, cola e entre } \\
\text { outras sustâncias. }\end{array}$ \\
\hline
\end{tabular}

Fonte: Artigos analisados (2016 e 2017).

O Quadro 4 demonstra os resultados de estudos relacionados ao adolescente, Segundo Patias, Silva e Aglio (2016). A exposição de adolescentes à violência aumenta a possibilidade de desenvolvimento de transtornos mentais e uso de substâncias psicoativas como álcool e drogas; os estudos de Tavares, et al., (2017). Apontam um maior predomínio do uso do álcool por adolescentes, por conseguinte uso de drogas como maconha, LSD, tabaco, loló, cocaína, ecstasy, cola e etc.

\subsection{Mulher}

Em relação ao adoecimento mental relacionado à mulher foram selecionadas quatro revisões sistemáticas aos quais abordaram aspectos da gestação, puerpério e condições de violência sexual, em que posteriormente todos esses aspectos 
colaboram para quadros de transtornos mentais em mulheres e encaixam essas mulheres em situações de risco ou vulnerabilidade (Quadro 5)

Quadro 5 - Resultados encontrados nos estudos de acordo com o título, autores, ano e principais considerações.

\begin{tabular}{|c|c|c|c|}
\hline TÍTULO & AUTORES & ANO & PRINCIPAIS CONSIDERAÇÕES \\
\hline 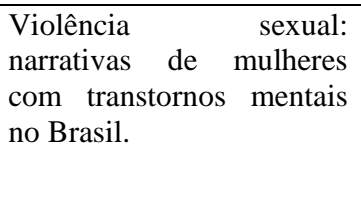 & $\begin{array}{ll}\text { Barbosa } & \& \\
\text { Souza } & \& \\
\text { Freitas. } & \end{array}$ & 2015 & $\begin{array}{l}\text { Identificou-se que as mulheres que participaram do estudo apresentaram baixa } \\
\text { autoestima e sentimento de inferioridade decorrentes da exclusão social que } \\
\text { vivenciaram por terem sido violentadas sexualmente. Ademais, foram } \\
\text { apresentados diagnósticos de transtorno afetivo bipolar, transtornos } \\
\text { esquizoafetivos ou delirantes, transtornos por uso de álcool e drogas, retardo } \\
\text { mental e demência precoce. }\end{array}$ \\
\hline $\begin{array}{l}\text { Fatores associados à } \\
\text { probabilidade de } \\
\text { transtorno mental comum } \\
\text { em gestante: estudo } \\
\text { transversal. }\end{array}$ & $\begin{array}{l}\text { Lucchese, } \\
\text { et al., }\end{array}$ & 2017 & $\begin{array}{l}\text { O estudo realizado com mulheres gestantes coletou dados, em que há uma alta } \\
\text { prevalência de transtorno mental comum em mulheres grávidas. Ademais, } \\
\text { verificou-se sintomas de ansiedade e depressão relacionado mais a mulheres } \\
\text { jovens com idade de } 17 \text { e } 29 \text { anos, casadas. }\end{array}$ \\
\hline $\begin{array}{l}\text { Evidências de } \\
\text { Sintomatologia Depressiva } \\
\text { no Pós-Parto Imediato. }\end{array}$ & $\begin{array}{l}\text { Monteiro, } \\
\text { et al., }\end{array}$ & 2018 & $\begin{array}{l}\text { O estudo encontrou alterações emocionais e fisiológicas que podem predispor } \\
\text { ao surgimento de quadros depressivos, aos quais foram encontrados esses } \\
\text { dados em } 24,5 \% \text { das mulheres participantes da pesquisa. Ademais, foram } \\
\text { coletados dados em que no Brasil há uma prevalência da sintomatologia de } \\
\text { Depressão Pós-Parto pode variar entre } 7,2 \% \text { e } 42,8 \% \text {. }\end{array}$ \\
\hline $\begin{array}{l}\text { Aspectos da gestação e } \\
\text { puerpério de mulheres } \\
\text { com transtornos mentais. }\end{array}$ & $\begin{array}{l}\text { Teixeira, et } \\
\text { al., }\end{array}$ & 2019 & $\begin{array}{l}\text { Observou-se predominância de quadros psicóticos agudos em gestantes, } \\
\text { também os transtornos mentais comuns (TMC) com sintomas de fadiga e } \\
\text { sinais depressivos, alta prevalência de TMC relacionado à autoestima feminina } \\
\text { e depressão pós-parto. }\end{array}$ \\
\hline
\end{tabular}

Fonte: Artigos analisados $(2015,2017,2018$ e 2019).

Já o Quadro 5 aponta os resultados de estudos relacionados à transtornos mentais em mulheres, de acordo com Barbosa, Souza e Freitas, (2015). Mulheres que sofreram violência sexual apresentam baixa autoestima e sentimento de inferioridade, também há predominância de transtorno de bipolaridade, esquizoafetivo, delirante e demência precoce. Nos estudos de Lucchese, et al., 2017. Detectaram uma maior prevalência de transtorno mental comum em gestantes, além do desenvolvimento de depressão e ansiedade em mulheres jovens e casadas. De acordo com Monteiro, et al., 2018. Há uma prevalência da sintomatologia de Depressão Pós-Parto pode variar entre 7,2\% e 42,8\% em puérperas. Já os estudos de Teixeira, et al., 2019. Detecta predominância de quadros psicóticos agudos em gestantes e TMC relacionado à autoestima feminina e depressão pós-parto.

\section{Discussões}

4.1 TOC, problemas internalizantes e ansiedade materna, relacionados aos aparecimentos de transtornos mentais na criança

Os resultados obtidos através das análises do Quadro 1, baseado nos estudos de (Bozzini, et al., 2018), mostram transtornos obsessivo compulsivo (TOC) com aspectos de rigidez alimentar em crianças e em indivíduos adultos que acarretaram na infância o devido transtorno, e por não tratar o quadro foi agravando até a fase adulta ou se mostrando presente. Desse modo, comparando esses resultados, deve-se ressaltar a presença de resultados semelhantes na presente pesquisa em os indivíduos que apresentaram quadro de TOC com a rigorosidade alimentar, seja a recusa da comida por questões de textura, sons no ato da alimentação que geravam incomodo e acerca da limpeza do alimento.

Além disso, perante análises do Quadro 2 e seus devidos resultados, aos quais foram baseados em (Lins \& Alvarenga, 2015) é notável a presença de problemas internalizantes sejam estes de quadros de ansiedade, retraimento, depressão, queixas somáticas e reatividade emocional. Partindo desse ponto, de modo fazendo uma avaliação dos dados do quadro 2 , é observável 
um nível maior de crianças com problemas internalizantes correlacionados em relação à vivência das mesmas com os pais em que estavam relativos à uma relação de controle crítico para aquela criança, ou mesmo, a situações hostis.

Rees, et al. (2019) evidenciam os efeitos da ansiedade materna sobre problemas emocionais na criança em que se apresentavam seja por um nível mais elevado ao aparecimento de transtornos, ou ansiedade materna relacionada a fobia social e sua relação com o apego seguro ou inseguro na criança. Logo, comparando os resultados do Quadro 3, é importante considerar que o período de pré-natal se torna um grande contribuinte para a saúde e desenvolvimento infantil daquela criança ao qual está sendo gerada, mas que, em contrapartida, o surgimento de transtornos na criança pode não só estar associados a exposição da mãe durante o período pré-natal a quadros de ansiedade e que para um diagnóstico efetivo devem analisar também o histórico familiar, o ambiente familiar, aspectos sociodemográficos e entre outros fatores.

\subsection{Aparecimento de transtornos mentais em adolescentes expostos a violência e vulneráveis para o uso de drogas}

Em relação aos estudos de Patias, et al., 2016. O autor evidencia a exposição a violência durante a adolescência relacionada com a saúde mental do indivíduo e, o quanto a mesma influencia seja como possíveis causas de aparecimento de transtornos em adolescentes, no que na maioria dos casos ocorrem dentro do ambiente familiar, e/ou mesmo, em situações corriqueiras seja na escola ou no grupo de amigos. Em contrapartida, discordando com o autor parcialmente, deve-se levar em consideração não só a exposição a violência como principal causa, mas também que ela age conjuntamente com o as mudanças hormonais e fisiológicas cujo aquele indivíduo está sofrendo, em que assim colaboram para o aparecimento de transtornos.

Em suma, comparando os resultados do quadro 4, é importante ressaltar uma semelhança aos quais tanto a exposição a violência quanto a vulnerabilidade para o uso de drogas colaboram para o aparecimento de transtornos no adolescente, em que apresentam em maioria dos casos a principal causa dentro de uma problemática no ambiente familiar. Partindo desse ponto, quase sempre os casos positivos para o aparecimento de adoecimento mental durante essa fase da vida se apresentavam por uma exposição a violência dentre os familiares, ou, o uso de drogas como modo de fuga dos problemas na família.

\subsection{Mulheres que se encontram em vulnerabilidade e o acarretamento de transtornos posteriormente}

Visto uma semelhança entre os estudos de (Barbosa, et al., 2015), (Lucchese, et al., 2017), (Monteiro, et al., 2018) e (Teixeira, et al., 2019), é observável transtornos relacionados em mulheres no pós-parto, em que há uma quantidade maior de casos de TMC (transtorno mental comum) em puérperas aos quais, é importante ressaltar que esse tipo de transtorno se relaciona a questões de saúde mental do paciente que não completa todos os fatores para se fechar um diagnóstico de ansiedade ou depressão. Ainda mais, há uma prevalência também de quadros de sintomatologias de depressão pós-parto e ansiedade entre os resultados de transtornos relacionados à puérperas.

Desse modo, de acordo com os resultados do quadro 5 da presente pesquisa, deve-se analisar o quanto a prevalência de TMC, depressão pós-parto e ansiedade em mulheres puérperas estão associados a questões da relação com o cônjuge, ou o fato de não conseguir lidar com opiniões sobre os cuidados com a prole, questões de gravidez indesejada, o não aceitação com as modificações no corpo que acontecem durante a gestação e após e, fatores de expectativas de pós-parto aos quais não ocorreram. Logo, devido uma análise dos resultados, também deve-se levar em consideração os fatores hormonais no pós-parto em que se comparados com o processo de adolescência, ambos possuem uma explosão de mudanças fisiológicas com consequência de uma série de hormônios aos quais influenciam também para casos transtornos, se associados a questões de vulnerabilidade e não tratar o adoecimento mental o quanto antes.

Em relação aos resultados de Barbosa \& Souza \& Freitas (2015) e os transtornos em mulheres que estiveram em situações de risco e vítimas de violência sexual, as evidências mostraram quadros de casos de traumas, transtornos mais complexos e consequências psicológicas nas vítimas. De certo, questões como essa se relacionam com o fato de que as vítimas 
não denunciaram o ato em que em maioria dos casos ocorreram em âmbito familiar, o que colaborou para questões de sentimentos de fracasso, impotência, falta de autonomia para o ato da denúncia e a não superação do ocorrido.

Concordando com o autor, com base nos dados colhidos é importante ressaltar o quanto as consequências dos atos citados no parágrafo anterior se apresentaram de forma com uma prevalência de quadros de pioras de transtornos de mulheres vítimas de violência sexual, aos quais associados ao trauma, também houve preconceito da sociedade com a vítima, em que neste caso colaboram exclusivamente para aspectos negativos de piora no adoecimento mental do indivíduo.

\section{Considerações Finais}

Em síntese os estudos apontam o quanto questões de vulnerabilidade e situações de risco em crianças, adolescentes e mulheres colaboram consequentemente para quadros de adoecimento mental nos mesmos. Em razão disso, deve-se levar em consideração que em maioria dos casos de transtornos mentais em crianças não ocorrem um tratamento efetivo aos quais o mesmo quadro de adoecimento mental foi prorrogado até a fase adulta, como por exemplo: o TOC relacionado à rigorosidade alimentar.

Por outro lado, há uma série de dados aos quais relatam o âmbito familiar influencia para o aparecimento e/ou prevenção de transtornos após a um determinado fator de vulnerabilidade. Logo, o mesmo apareceu relacionado aos três grupos estudados, mas que em contrapartida deve levar em consideração outras questões contribuintes para a saúde e adoecimento mental da população estudada, como: fatores genéticos, sociodemográficos, sociais e ambientais.

Em suma é evidente, portanto, o quanto é importante o papel da equipe multiprofissional de saúde juntamente com a família para quadros de melhora em questões de adoecimento mental em indivíduos que estão vulneráveis ou em risco. Ademais, destaca-se também o quanto essa temática e situações de adoecimento mental devem ser reavaliadas e com uma visão mais ampliada perante a sociedade, em que os transtornos mentais deveriam ser considerados e tratados com uma maior importância.

Por fim, conclui-se que há uma necessidade de ampliação de estudos sobre o tema abordado ao qual, dessa forma contribuirá para melhores resultados e, consequentemente compreensão acerca os transtornos mentais relacionados à criança, adolescente e mulher. Dessa maneira, tanto os profissionais, como também a família das vítimas de adoecimento mental acarretaria uma melhora na atuação de atendimento e suporte ao indivíduo com transtorno.

\section{Referências}

Azevedo, R. C. S., \& Barros, M. B. A., \& Senicato, C. (2018) Transtorno mental comum em mulheres adultas: identificando os segmentos mais vulneráveis. Cien Saude Colet. https://www.scielo.br/j/csc/a/rSxF9pjYHk5MwQ3xrvS5zcT/?format=html. https://doi.org/10.1590/1413-81232018238.13652016.

Barbosa, J. A. G., \& de Souza, M. C. M. R.., \& Freitas, M. I. F., (2015). Violência sexual: narrativas de mulheres com transtornos mentais no Brasil. Rev Panam Salud Publica. https://assets-compromissoeatitude-ipg.sfo2.digitaloceanspaces.com/2015/07/BARBOSAeoutras2015.pdf

Barbosa, L. N. F., \& Asfora, G. C. A., \& Moura, M. C., (2020). Ansiedade e depressão e uso de substâncias psicoativas em jovens universitários. SMAD, Rev. Eletrônica Saúde Mental Álcool Drog. vol.16. http://pepsic.bvsalud.org/scielo.php?script=sci_arttext\&pid=S1806-69762020000100014. http://dx.doi.org/10.11606/issn.1806-6976.smad.2020.155334.

Bozzini, A. B, et al., 2018. O pediatra deve investigar sintomas de transtornos obsessivos compulsivos em crianças com dificuldades alimentares? Rev. Paul.Pediatr. https://www.scielo.br/j/rpp/a/dhNprxxgkJTtNGRZZXH78dk/?lang=pt. https://doi.org/10.1590/1984-0462/;2019;37;1;00010

Duko, B., \& Wolde, D., \& Alemayehu, Y. (2020). A epidemiologia da depressão pós-parto na Etiópia: uma revisão sistemática e meta-análise. Reprod Health 17, 180 (2020). https://doi.org/10.1186/s12978-020-01035-1.

Kirsch, D. E., et al, (2021). Maus-tratos na infância, volume de substância cinzenta paralímbica pré-frontal e uso de substâncias em adultos jovens e interações com risco de transtorno bipolar. Sci Rep 11, 123. https://doi.org/10.1038/s41598-020-80407-w.

Lins, T, et al., (2015). Controle Psicológico Materno e Problemas Internalizantes em Pré-Escolares. Psic.: Teor. e Pesq., Bahia,. http://dx.doi.org/10.1590/0102-37722015032092311319 
Research, Society and Development, v. 10, n. 14, e427101421987, 2021

(CC BY 4.0) | ISSN 2525-3409 | DOI: http://dx.doi.org/10.33448/rsd-v10i14.21987

Luciano, M, et al., (2017). Perfil de adolescentes e vulnerabilidade para o uso de álcool e outras drogas. Revista de Enfermagem UFPE on line, Recife. https://periodicos.ufpe.br/revistas/revistaenfermagem/article/view/22571/24345. 10.5205/reuol.12834-30982-1-SM.1110201727

Lucchese, R, et al., (2017). Fatores associados à probabilidade de transtorno mental comum em gestante: um estudo transversal. https://www.scielo.br/j/ean/a/J6kDshGC6KHmDn8MHNW48mD/?lang=pt. https://doi.org/10.1590/2177-9465-EAN-2016-0094.

Medeiros, M. P., \& Zannello, V. (2018). Relação entre a violência e a saúde mental das mulheres no Brasil: análise das políticas públicas. https://www.epublicacoes.uerj.br/index.php/revispsi/article/view/38128/26768.

Mendonça, M. F. S., \& Ludemir, A. B. (2017). Violência por parceiro íntimo e incidência de transtorno mental comum. Rev Saude Publica, https://www.scielo.br/j/rsp/a/pz3g38HCG5nG94pxpks7z4B/?lang=pt. https://doi.org/10.1590/S1518-8787.2017051006912.

Molina, M. L, et al., (2019). Problemas emocionais e comportamentais: um estudo baseado na escola no sul do Brasil. https://www.scielo.br/scielo.php?script=sci_arttext\&pid=S2237-60892019000300211\#B8. https://doi.org/10.1590/2237-6089-2017-0119

Monteiro, K. A, et al., (2018). Evidencias de sintomatologia depressiva no pós-parto imediato. Revista Brasileira De Ciências Da Saúde, v. 22. No 4. 379 388, 2018. 10.4034/RBCS.2018.22.04.12

Monteiro, D. S, et al., (2020). Fatores associados a transtorno mental comum em adolescentes escolares. Revista Brasileira de Enfermagem. https://www.scielo.br/j/reben/a/dSfCCJj434cdT3JSyHc7kBz/?lang=pt. https://doi.org/10.1590/0034-7167-2019-0847.

Patias, N., \& Silva, D., \& Aglio, D. (2016) Exposição de adolescentes à violência em diferentes contextos: relações com a saúde mental. Temas psicol. vol.24 no. 1 Ribeirão Preto mar. http://pepsic.bvsalud.org/scielo.php?script=sci_arttext\&pid=S1413-389X2016000100010\&lng=pt\&nrm=iso\&tlng=pt. http://dx.doi.org/10.9788/TP2016.1-14.

Rees, S., \& Channon, S., \& Waters, C.S. (2018). O impacto da ansiedade pré-natal e pós-natal materna nos problemas emocionais das crianças: uma revisão sistemática. Psiquiatria infantil e adolescente europeia. https://link.springer.com/article/10.1007/s00787-018-1173-5.

Sousa, C. M. S, et al., (2019). Ideação suicida e fatores associados entre escolares adolescentes. Revista de Saúde Pública. https://www.scielo.br/j/rsp/a/4nWHhmPNt9Zz9y8X49ZW5xc/?lang=pt. https://doi.org/10.11606/s1518-8787.2020054001637.

Tavares, M. L. O., \& Casarubi, L. E., \& Scher, C. R., (2019). Saude mental e cuidado de enfermagem em psiquiatria. SAGAH, 2019. Biblioteca Virtual BIBLIOTECA A.

Teixeira, C. S, et al., (2019). Aspectos da gestação e puerpério de mulheres com transtornos mentais. Rev enferm UFPE on line. 2019;13:e239705. https://pesquisa.bvsalud.org/portal/resource/pt/biblio-1052354. https://doi.org/10.5205/1981-8963.2019.239705.

Thiengo, D. S., \& Cavalcante, M. T., \& Lovisi, G. M. (2018). Prevalência de transtornos mentais entre crianças e adolescentes e fatores associados: uma revisão sistemática. https://www.scielo.br/scielo.php?script=sci_arttext\&pid=S0047-20852014000400360. https://doi.org/10.1590/0047-2085000000046 